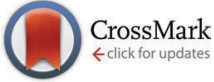

Cite this: New J. Chem., 2016, 40, 1057

Received (in Nottingham, UK) 1st May 2015,

Accepted 15th August 2015

DOI: $10.1039 / c 5 n j 01097 g$

www.rsc.org/njc

\title{
Autonomous movement induced in chemically powered active soft-oxometalates using dithionite as fuel $\dagger$
}

\author{
Apabrita Mallick, Dipti Lai and Soumyajit Roy*
}

Synthesis of autonomously moving nano or micromotors is an immediate challenge in current nanoscience and nanotechnology. In this work we report a system based on soft-oxometalates (SOMs) which is very easy to synthesize and moves autonomously in response to chemical stimuli like that of a reducing agent-dithionite. The redox active $\mathrm{Mo}^{\mathrm{VI}}$ sites of SOMs are used for oxidizing dithionite to generate $\mathrm{SO}_{2}$ to propel the micromotors. We explain this motion qualitatively and also show how surface interaction and adsorption isotherm of the evolved gas influence the power conversion efficiency of these micromotors.

\section{Introduction}

Synthesis of autonomously moving soft and active matter ${ }^{1}$ is an immediate challenge for modelling biological phenomenon. In recent times, although moving matter has been designed,$^{2-5}$ its synthesis is tedious. Here we report a system based on softoxometalates $^{6,7}$ (SOMs) which is very easy to synthesize and is used as a model system in our study. We now explore the current status in the field of micromotors.

The first well known synthetic motor ${ }^{8}$ was prepared by Whitesides et al. in 2002 which used Pt catalyst to drive millimetre scale plastic disks. Synthetic motors of micrometer scale were prepared in 2004-2005. ${ }^{9,10}$ These micromotors were bimetallic rods of $\mathrm{Pt}-\mathrm{Au}^{9}$ and $\mathrm{Ni}-\mathrm{Au}^{10}$ of length $2-3 \mu \mathrm{m}$ which catalytically decomposed $\mathrm{H}_{2} \mathrm{O}_{2}$ to $\mathrm{H}_{2} \mathrm{O}$ and $\mathrm{O}_{2}$ and moved with a speed of $\sim 10 \mu \mathrm{m} \mathrm{s}^{-1}$. The movement of synthetic micromotors is controlled by artificial stimuli such as chemical fuel, ${ }^{11-15}$ magnetic field, ${ }^{16-18}$ electric field, ${ }^{19-21}$ ultrasonic sound ${ }^{22}$ or light. ${ }^{23-25}$ Thus the operation of micromotors is dependent on the source of energy consumption and accordingly they can be classified as bubble propelled ${ }^{5,11,12}$ micromotors, self electrophoretically propelled ${ }^{13-15}$ micromotors, magnetically driven ${ }^{16-18}$ micromotors, electrically driven ${ }^{19-21}$ micromotors, ultrasound propelled ${ }^{22 a, b}$ micromotors and light driven ${ }^{23-25}$ micromotors. The movement ${ }^{26}$ of active motors can be in the form of translation, ${ }^{9}$ rotation, ${ }^{10}$ delivery ${ }^{27}$ or collective behaviour. ${ }^{24}$ Chemically

EFAML, Materials Science Centre, Department of Chemistry,

Indian Institute of Science Education and Research, Kolkata-741246, India.

E-mail: s.roy@iiserkol.ac.in

$\dagger$ Electronic supplementary information (ESI) available. See DOI: 10.1039/ c5nj01097g powered micromotors ${ }^{12}$ are mainly propelled due to the gas generated by the surface catalytic decomposition ${ }^{9}$ of the fuel. Chemically propelled micromotors can be either bubble propelled ${ }^{11}$ or self electrophoretically ${ }^{5}$ propelled. The requirement of chemical fuels can be obviated by using micromotors which use physical sources for their energy requirement like magnetic micromotors ${ }^{16}$ driven in a magnetic field that convert their rotary motion to axial translation. Biocompatible fuel free micromotors can also be obtained from ultrasonic acoustic waves. ${ }^{22}$ In the low Reynold's number regime, Brownian motion plays the dominating role and thus the autonomous movement of micro objects is restricted. ${ }^{26}$ At microscale, interfacial forces dominate over inertia ${ }^{28}$ which is exploited to devise micromotors.

Soft-charged metal oxide based structures, the soft-oxometalates ${ }^{6}$ (SOMs), with metals in a high oxidation state provide us with an opportunity to induce autonomous movement in these SOMs. SOMs have also been moved using optical forces before by us. ${ }^{23}$ The redox chemistry of SOM provides a possibility for generating a gas by the oxidation of a reductant which can act as a fuel and in turn propel the SOMs. Here we exploit this possibility. More precisely, we take the step of inducing autonomous movement in heptamolybdate SOMs with the help of the chemical fuel dithionite. We ask whether it is possible to exploit the redox active $\mathrm{Mo}^{\mathrm{VI}}$ sites on SOMs to generate $\mathrm{SO}_{2}$ gas by oxidation of the reductant dithionite. We address this question here.

\section{Results and discussion}

The chemistry of inducing motion in SOMs

For our study, we choose dithionite as the fuel and metal oxide (oxomolybdate) as the reacting surface. We note that the SOMs 


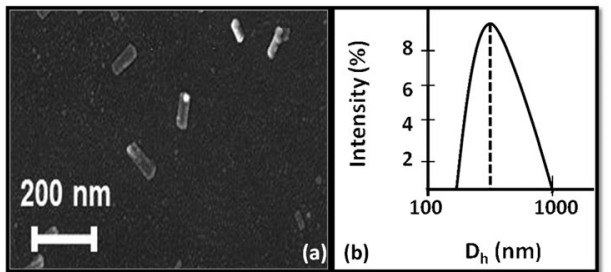

Fig. 1 (a) SEM image and (b) DLS size distribution plot of heptamolybdate SOMs.

are rod shaped in nature with their size in agreement with DLS and SEM results (see Fig. 1).

The length of SOM rods from SEM is around $200 \mathrm{~nm}$. From DLS the hydrodynamic diameter $\left(D_{\mathrm{h}}\right)$ is around $500 \mathrm{~nm}$ which is due to the tumbling motion of the rods that effectively gives rise to a sphere of $500 \mathrm{~nm}$ diameter.

The aqueous solution of sodium dithionite is unstable ${ }^{29}$ and in aerobic and acidic conditions (under the conditions of autonomous movement shown by the SOMs) it generates sulphur and sulphur dioxide. So we used freshly prepared solution of sodium dithionite for our experiment.

When an aqueous solution of sodium dithionite $\left(\mathrm{Na}_{2} \mathrm{~S}_{2} \mathrm{O}_{4}\right)$ is added to the dispersion of mildly acidic isopolyacids of SOMs, dithionite gets oxidised to $\mathrm{SO}_{2}$ and $\mathrm{Mo}^{\mathrm{VI}}$ centres of $\mathrm{SOM}$ get reduced to $\mathrm{Mo}^{\mathrm{V}}$ centres which ultimately generate molybdenum blue type SOMs and propels the SOMs. Thus propulsion of SOMs is the result of a redox reaction which gives rise to a gaseous oxidized product $\mathrm{SO}_{2}$ and a reduced molybdenum blue $\mathrm{SOM}$ which is blue in colour due to IVCT type transition $\left(\mathrm{Mo}^{\mathrm{V}} \rightarrow \mathrm{Mo}^{\mathrm{VI}}\right)$. The $\mathrm{SO}_{2}$ produced by the reaction of dithionite with heptamolybdate remains in the solution for a considerable amount of time which in turn propels these active SOM motors. So, the lifetime of active
SOMs is also quite significant, though not infinite. In this reaction $\mathrm{Mo}^{\mathrm{VI}}$ oxidises ${ }^{30}$ dithionite to $\mathrm{SO}_{2}$ in accordance with the following reaction (shown schematically):

$$
\mathrm{Mo}_{7}^{\mathrm{VI}} \mathrm{O}_{24}+\mathrm{S}_{2} \mathrm{O}_{4}{ }^{2-} \rightarrow \text { molybdenum blue }+2 \mathrm{SO}_{2}(\mathrm{~g})
$$

This redox process in fact causes the motion of the SOMs oxidizing the dithionite and reducing the SOMs. The propulsion of SOMs due to the oxidation of $\mathrm{SO}_{2}$ is coupled with the reduction of SOMs and thus the emergence of $\mathrm{Mo}^{\mathrm{V}} \rightarrow \mathrm{Mo}^{\mathrm{VI}}$ intervalence charge transfer (IVCT) in the molybdenum blue SOMs; the extent of $\mathrm{SO}_{2}$ evolution can hence be monitored by electronic absorption spectroscopy (Fig. 2). The extent of $\mathrm{SO}_{2}$ produced in the above reaction is coupled with the generation of blue SOMs which is signalled by the intensity of the $\mathrm{Mo}^{\mathrm{VI}} \rightarrow \mathrm{Mo}^{\mathrm{V}}$ IVCT band in blue SOMs. In this way we are able to find the extent of generation of $\mathrm{SO}_{2}$ from electronic absorption spectroscopy and we plot the amount of $\mathrm{SO}_{2}$ liberated against the loading variation of dithionite used for propelling the SOM micromotors (Fig. 3).

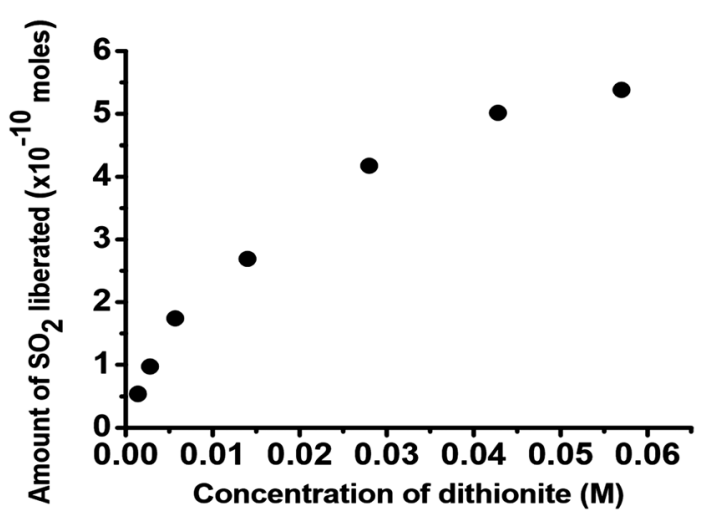

Fig. 3 Plot of amount of $\mathrm{SO}_{2}$ liberated vs. loading variation of dithionite.
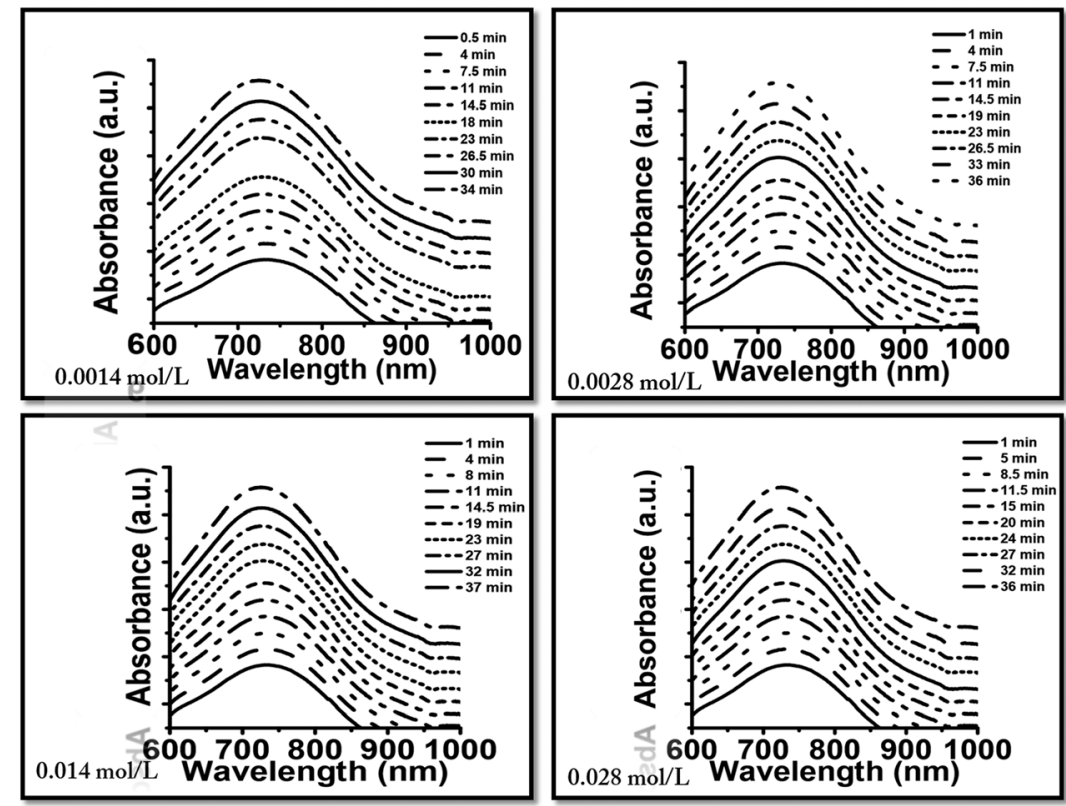

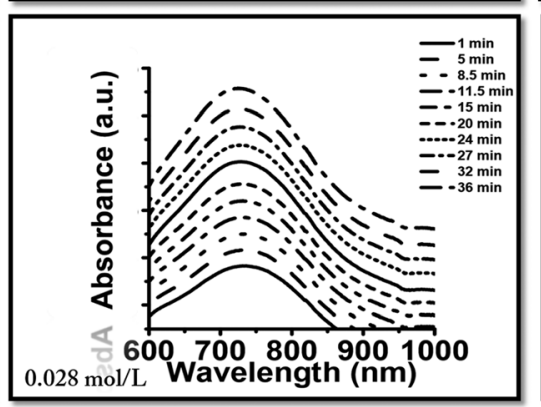

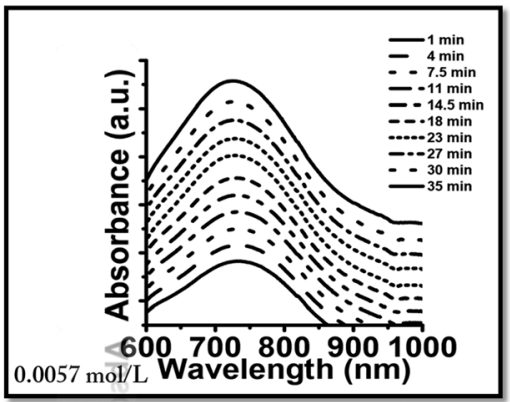

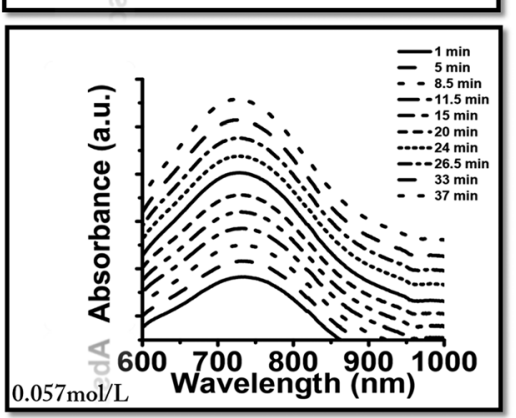

Fig. 2 UV-visible spectra of the SOM dithionite system at different concentrations of dithionite. 


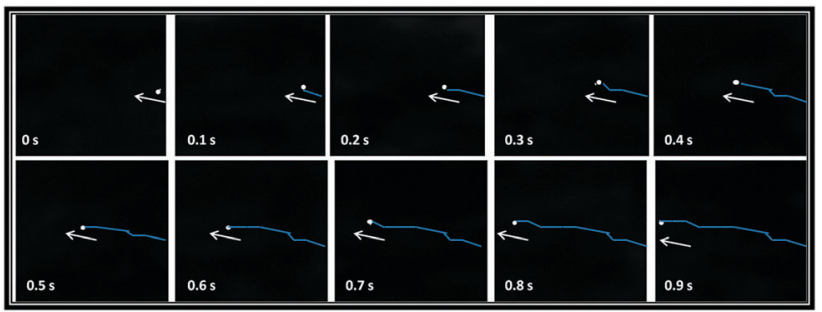

Fig. 4 Time lapse images of moving SOM in $0.014 \mathrm{~mol} \mathrm{~L}^{-1}$ sodium dithionite. The direction of motion is shown by the arrow and the blue lines indicate the trace lines.

On the other hand motion is induced in SOMs (Fig. 4 and ESI $\dagger$ ) due to the movement of the osmotic boundary between the reacting interface and the moving particle. To understand the effect of the fuel dithionite qualitatively, we load sodium dithionite of varying concentrations in the system. To analyse the individual movement of SOM particles we calculate the velocity of SOM at different concentrations of dithionite (Fig. 5(a)) and also determine the trajectories using ImageJ and TrackPy ${ }^{31}$ (Fig. 5(b)-(g)). The details of analysis are given in the experimental section.

The movement of SOMs in aqueous dispersion is entirely Brownian in nature. On addition of the fuel, reaction starts at the surface of the SOM and it shows directed autonomous translation. With the increase in the concentration of the fuel, the velocity of SOMs increases initially up to 52 body lengths $\mathrm{s}^{-1}$ (for $0.014 \mathrm{~mol} \mathrm{~L}^{-1}$ of sodium dithionite). But after that the velocity decreases even if the dithionite concentration is increased. At $0.0574 \mathrm{~mol} \mathrm{~L}^{-1}$ dithionite concentration the motion of SOMs is again Brownian in nature. Above $0.0574 \mathrm{~mol} \mathrm{~L}^{-1}$ we cannot track the movement of active SOMs because the colour of the dispersion becomes too dark (blue) for microscopic imaging. We demonstrate this graph of velocity of active SOMs and the trajectories in Fig. 5.

On the motion of SOMs. We now explain qualitatively the mechanism of the induced motion of SOMs. As the above reactions start, a mobile diffuse boundary separating the starting SOM with the molybdenum blue type species' interface is generated. This interface generates an osmotic boundary. At this boundary $\mathrm{SO}_{2}$ gas is produced due to which a slip velocity is created between the SOM interface and the continuous medium, which propels the SOMs.

As we increase the fuel concentration, more and more sodium dithionite reacts with SOMs and more $\mathrm{SO}_{2}$ is produced. The thrust produced by the evolved gas on SOM increases. Consequently, SOM travels with increasing velocity till it reaches a tipping point. When more gas is evolved, more $\mathrm{SO}_{2}$ molecules get adsorbed on the surface of the SOM. Thus the unreacted SOM interface now interacts with a less amount of fuel and we observe a decrease in the velocity of SOMs. For sodium dithionite the tipping point is observed at $0.014 \mathrm{~mol} \mathrm{~L}^{-1}$ concentration. Beyond this concentration of $0.014 \mathrm{~mol} \mathrm{~L}^{-1}$ of sodium dithionite, we believe that the SOM surface sites are all saturated and this creates an additional viscous drag and consequently the velocity of SOMs drops. With further reaction, the osmotic boundary reaches equilibrium and there is no asymmetry between the osmotic boundary and the SOM surface. Thus the slip velocity decreases to near zero. Since all the reactions were carried out at a uniform temperature we can plot adsorption isotherm by calculating the area of SOM occupied by adsorbed $\mathrm{SO}_{2}$ where $\theta$ is the fraction of the surface that is covered with gaseous molecules and $p$ is the pressure of $\mathrm{SO}_{2}$ gas.

From the graph (Fig. 6) it is evident that the fraction of occupied sites on active SOM surface increases with the increasing pressure

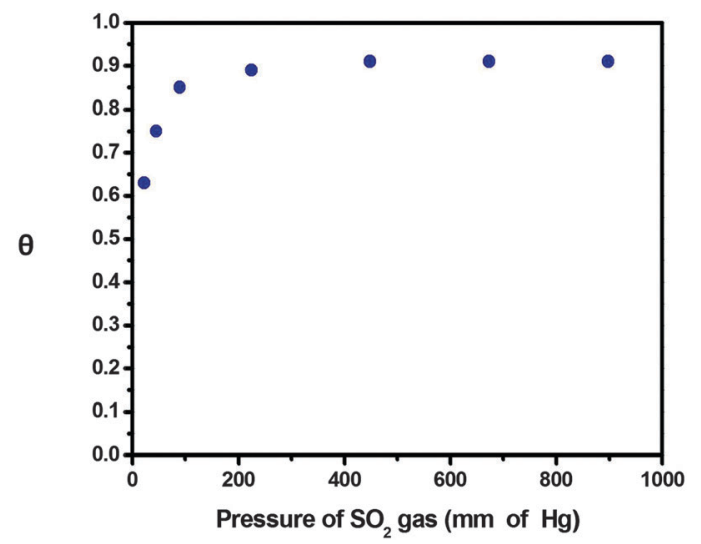

Fig. 6 Adsorption isotherm of evolved $\mathrm{SO}_{2}$ on the surface of SOMs.

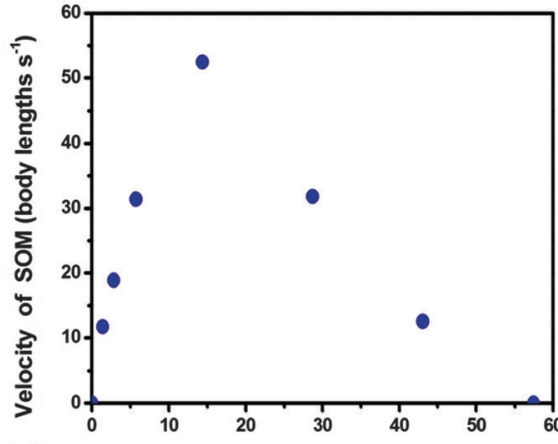

(a) Concentration of sodium dithionite $\left(10^{-3} \mathrm{~mol} / \mathrm{L}\right)$
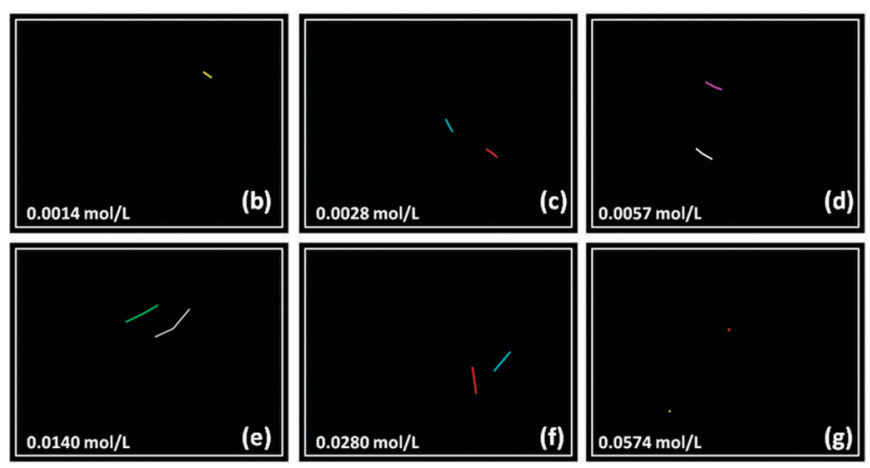

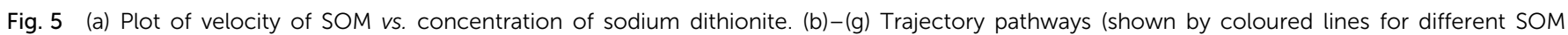
particles) of moving SOM in different dithionite concentrations for 1 second. 
Table 1 Surface interaction between the product of catalytic decomposition and SOM surface

\begin{tabular}{ll}
\hline Concentration of sodium dithionite $\left(\mathrm{mol} \mathrm{L}^{-1}\right)$ & $\alpha_{\mathrm{eff}}\left(\mathrm{nm}^{2}\right)$ \\
\hline 0.0014 & 3.46 \\
0.0028 & 2.78 \\
0.0057 & 2.31 \\
0.0140 & 1.54 \\
0.0280 & 0.46 \\
0.0430 & 0.12
\end{tabular}

of $\mathrm{SO}_{2}$. On increasing the concentration of dithionite, more $\mathrm{SO}_{2}$ gas is produced and the pressure of $\mathrm{SO}_{2}$ also increases as pressure is directly proportional to concentration. When all the active sites on SOM are occupied by the $\mathrm{SO}_{2}$ gas $\theta$ reaches a saturation point.

We can describe the motion of SOMs using osmophoresis. In this model, for the reaction rate dominated regime, ${ }^{32}$ the average velocity of the particles is given by

$$
\nu=-\frac{\Gamma k_{\beta} T \alpha_{\mathrm{eff}}}{8 \pi D R^{2} \eta}
$$

where $\Gamma$ is the reaction rate, $k_{\beta} T$ is the thermal energy, $\alpha_{\mathrm{eff}}$ describes the interaction between the molybdenum blue formed and the unreacted SOM surface, $D$ is the diffusivity, $R$ is the hydrodynamic radius of the particle and $\eta$ is the coefficient of viscosity. $\alpha_{\text {eff }}$ is very small and lies in the range $0.12 \mathrm{~nm}^{2}$ to $3.46 \mathrm{~nm}^{2}$ (Table 1 ).

On power conversion efficiency. The power conversion efficiency $^{33}$ of these micromotors can be defined as

$$
\eta=\frac{P_{\text {mech }}}{P_{\text {chem }}}
$$

where $P_{\text {mech }}$ is the mechanical energy output and is given by $P_{\text {mech }}=F_{\text {drag }} \nu=f \nu^{2}$ and $P_{\text {chem }}$ is the chemical energy input and is given by $P_{\text {chem }}=n \Delta_{\mathrm{r}} G$.

$$
\text { For cylinders, } f=\frac{2 \pi \mu L}{\ln \left(\frac{L}{R}\right)-0.72} \text {. }
$$

Here $F_{\text {drag }}$ is the drag force on the cylindrical SOM, $f$ is the drag coefficient, $\mu$ is the dynamic viscosity of water, $L$ is the length of $\mathrm{SOM}, R$ is its radius, $\nu$ is the motor speed, $n$ is the gas evolution rate in units of mol per (SOM s) and $\Delta_{\mathrm{r}} G$ is the Gibbs free energy of the decomposition of dithionite. We calculate the values of $P_{\text {mech }}$ and $P_{\text {chem }}$ and find the energy efficiency of SOM micromotors (Table 2).

The power conversion efficiency (Fig. 7) of SOM micromotors using dithionite as the fuel varies in the order of $10^{-12}$ to $10^{-10}$.

Table 2 Power conversion efficiency of the SOM micromotors in the SOM-dithionite system

\begin{tabular}{llll}
$\begin{array}{l}\text { Concentration of } \\
\text { sodium dithionite } \\
\left(\mathrm{mol} \mathrm{L}^{-1}\right)\end{array}$ & $P_{\text {mech }}$ (Joules) & $P_{\text {chem }}($ Joules) & Efficiency $(\eta)$ \\
\hline 0.0014 & $2.14 \times 10^{-22}$ & $8.58 \times 10^{-13}$ & $2.49 \times 10^{-10}$ \\
0.0028 & $5.56 \times 10^{-22}$ & $1.71 \times 10^{-12}$ & $3.25 \times 10^{-10}$ \\
0.0057 & $7.15 \times 10^{-22}$ & $3.43 \times 10^{-12}$ & $2.08 \times 10^{-10}$ \\
0.0140 & $4.30 \times 10^{-21}$ & $8.58 \times 10^{-12}$ & $5.01 \times 10^{-10}$ \\
0.0280 & $1.58 \times 10^{-21}$ & $1.71 \times 10^{-11}$ & $9.29 \times 10^{-11}$ \\
0.0430 & $2.45 \times 10^{-22}$ & $2.57 \times 10^{-11}$ & $9.53 \times 10^{-12}$
\end{tabular}

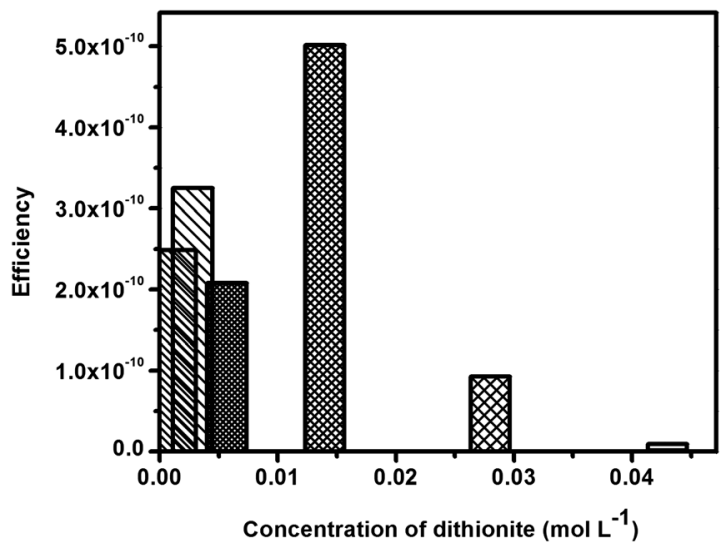

Fig. 7 Power conversion efficiency of SOM micromotors at different dithionite concentrations.

The maximum efficiency is observed to be $5.01 \times 10^{-10}$ for $0.014 \mathrm{~mol} \mathrm{~L}^{-1}$ dithionite where the velocity of these SOM particles is also maximum.

\section{Conclusion}

To conclude, using molybdenum based soft-oxometalates (SOMs) and employing sodium dithionite as a fuel, we can make SOMs move. The motility of SOMs stems from the evolution of the $\mathrm{SO}_{2}$ gas by the oxidation of dithionite to $\mathrm{SO}_{2}$ with the concomitant reduction of $\mathrm{Mo}_{7}-\mathrm{SOMs}$ to molybdenum blue type SOMs. The reaction creates an osmotic boundary between the newly generated product (including the gas) and the unreacted reactant SOM surface. The chemical potential gradient between the SOM surface and the reactive osmotic interface (with evolving gas) gives rise to a slip velocity to the SOMs propelling them. The velocity of SOMs increases up to a certain limit ( 52 body lengths $\mathrm{s}^{-1}$ ) till the SOM surface is saturated with evolved gases as seen from adsorption isotherm. At such saturation value, the SOM velocity reaches a maximum of 52 body lengths $\mathrm{s}^{-1}$ and due to the viscous drag induced by the evolved gases on the SOM surface, the velocity decreases and finally drops to zero, when the viscous drag annuls the slip velocity. This explains that using a simple redox system and exploiting this potential in SOMs it is possible to construct SOM micromotors and in principle possibilities exist for fine-tuning their motion. The high propulsion velocity and facile synthesis of heptamolybdate SOM hold considerable importance in the field of active matter and can be used in future for cargo transport, ${ }^{34}$ catalysis $^{35}$ and drug release. ${ }^{36}$ A more quantitative understanding of this phenomenon using other model systems is currently under investigation in our laboratory.

\section{Experimental details}

\section{Preparations}

The reagents were purchased from commercial sources (Merck) and used without further purification. The glassware was cleaned in an acid bath, base bath and rinsed with isopropanol followed by acetone and kept in an oven for 48 hours prior to use. 
Synthesis of ammonium heptamolybdate soft oxometalate (SOM). Ammonium heptamolybdate tetrahydrate $(1500 \mathrm{mg}$, $1.213 \mathrm{mmol}$ ) was dissolved in distilled water $(4 \mathrm{~mL})$ and heated until simmering hot. A clear dispersion of ammonium heptamolybdate was formed, which was then stored in a refrigerator for 10 minutes. The dispersion was brought to room temperature which scattered light from a laser.

Synthesis of sodium dithionite solutions. Calculated amounts of sodium dithionite were dissolved in $10 \mathrm{~mL}$ of distilled water to prepare dithionite solutions of concentrations $0.0014 \mathrm{~mol} \mathrm{~L}^{-1}$, $0.0028 \mathrm{~mol} \mathrm{~L}^{-1}, 0.0057 \mathrm{~mol} \mathrm{~L}^{-1}, 0.0140 \mathrm{~mol} \mathrm{~L}^{-1}, 0.0280 \mathrm{~mol} \mathrm{~L}^{-1}$, $0.0430 \mathrm{~mol} \mathrm{~L}^{-1}$ and $0.0574 \mathrm{~mol} \mathrm{~L}^{-1}$.

\section{Instrumental analysis}

Microscopy using an inverted fluorescence microscope. An Olympus IX81 epifluorescence microscope with a motorized stage was used for recording videos. A $22 \times 40$ cover slip was cleaned with methanol and dried to remove any unwanted adsorbed material. The SOM dispersion $(10 \mu \mathrm{L})$ was placed on that cover slip. The cover slip was then placed on the microscope scanning stage and the stage was controlled using a joystick. $10 \mu \mathrm{L}$ of sodium dithionite solution (of known concentration) was added to the SOM dispersion using a $10 \mu \mathrm{L}$ micropipette. The dispersion which was initially colourless turned blue on addition of dithionite. The microscope was focused at $40 \times$ objective and the videos were recorded using a DSIC camera at a rate of 10 frames per second.

Characterisation using scanning electron microscopy (SEM). The SEM image was taken on a SUPRA 55 VP-41-32 instrument with the SmartSEM version 5.05 Zeiss software.

Characterisation using dynamic light scattering (DLS) measurements. $0.5 \mathrm{~mL}$ of heptamolybdate SOM dispersion was diluted with $10 \mathrm{~mL}$ of deionised water. This dispersion was irradiated with a hand held laser pointer of wavelength $635 \mathrm{~nm}$ which resulted in the scattering of a single well-defined line. The resulting dispersion was then subjected to DLS experiment using a Malvern Zetasizer.

\section{Image analysis}

Analysis with ImageJ. The raw image sequence in .TIFF format was converted to .AVI form using ImageJ. From these videos each frame was separately taken and the SOM particles in that frame were analysed manually. That is, the coordinates of the position of a SOM particle were measured over 3 frames, the body length of that particular SOM was taken into account (as SOMs are of varying size), time gap between the frames was noted and using these the velocity of each SOM was calculated. This procedure was repeated for a number of SOMs in each video corresponding to a particular dithionite concentration. The average of these velocities was considered as the velocity of the SOM at that particular dithionite concentration. The same procedure was carried out for all dithionite concentrations. The velocities of SOM were plotted in a graph against concentration of sodium dithionite.

Analysis with Trackpy. Trackpy is a python packaging tool used for particle tracking. Using this code each video was analysed and trajectories of SOM were obtained. Trackpy first identified SOM particles in each frame with selective filters in it and then connected the frames in which a SOM particle was present to obtain the particle trajectories. For each dithionite concentration the code was written separately and particle trajectories were obtained.

\section{Acknowledgements}

SR gratefully acknowledges the grants from IISER-Kolkata, India, DST-fast track, BRNS-DAE grant, Mr Ritabrata Ghosh for helping with the imaging and Mr Abhrajit Laskar (IMSc, Chennai) for his help and support. The authors thank Prof. Ronojoy Adhikari (IMSc, Chennai) for helpful discussion.

\section{References}

1 S. Ghose and R. Adhikari, Phys. Rev. Lett., 2014, 112, 118102.

2 S. Sengupta, D. Patra, I. Ortiz-Rivera, A. Agrawal, S. Shklyaev, K. K. Dey, U. Córdova-Figueroa, T. E. Mallouk and A. Sen, Nat. Chem., 2014, 6, 415-422.

3 J. Li, W. Gao, R. Dong, A. Pei, S. Sattayasamitsathit and J. Wang, Nat. Commun., 2014, 5, 5026.

4 K. Kim, X. Xu, J. Guo and D. Fan, Nat. Commun., 2014, 5, 3632. 5 S. Sanchez, A. N. Ananth, V. M. Fomin, M. Viehrig and O. G. Schmidt, J. Am. Chem. Soc., 2011, 133, 14860-14863.

6 S. Roy, CrystEngComm, 2014, 16, 4667-4676.

7 S. Roy, Comments Inorg. Chem., 2011, 32, 113.

8 R. F. Ismagilov, A. Schwartz, N. Bowden and G. M. Whitesides, Angew. Chem., 2002, 114, 674-676.

9 W. F. Paxton, K. C. Kistler, C. C. Olmeda, A. Sen, S. K. S. Angelo, Y. Cao, T. E. Mallouk, P. E. Lammert and V. H. Crespi, J. Am. Chem. Soc., 2004, 126, 13424-13431.

10 S. Fournier-Bidoz, A. C. Arsenault, I. Manners and G. A. Ozin, Chem. Commun., 2005, 441-443.

11 J. Wang and W. Gao, ACS Nano, 2012, 6, 5745-5751.

12 W. Gao, S. Sattayasamitsathit, J. Orozco and J. Wang, J. Am. Chem. Soc., 2011, 133, 11862-11864.

13 S. Sattayasamitsathit, W. Gao, P. Calvo-Marzal, K. M. Manesh and J. Wang, ChemPhysChem, 2010, 11, 2802-2805.

14 R. Laocharoensuk, J. Burdick and J. Wang, ACS Nano, 2008, 2, 1069-1075.

15 N. S. Zacharia, Z. S. Sadeq and G. A. Ozin, Chem. Commun., 2009, 5856-5858.

16 L. Zhang, T. Petit, Y. Lu, B. E. Kratochvil, K. E. Peyer, R. Pei, J. Lou and B. J. Nelson, ACS Nano, 2010, 4, 6228-6234.

17 A. Ghosh and P. Fischer, Nano Lett., 2009, 9, 2243-2245.

18 S. Tottori, L. Zhang, F. Qiu, K. K. Krawczyk, A. FrancoObregón and B. J. Nelson, Adv. Mater., 2012, 24, 811-816.

19 G. Loget and A. Kuhn, J. Am. Chem. Soc., 2010, 1, 15918-15919.

20 P. Calvo-Marzal, S. Sattayasamitsathit, S. Balasubramanian, J. R. Windmiller, C. Dao and J. Wang, Chem. Commun., 2010, 46, 1623-1624.

21 S. T. Chang, V. N. Paunov, D. N. Petsev and O. D. Velev, Nat. Mater., 2007, 6, 235-240. 
22 (a) V. Garcia-Gradilla, J. Orozco, S. Sattayasamitsathit, F. Soto, F. Kuralay, A. Pourazary, A. Katzenberg, W. Gao, Y. Shen and J. Wang, ACS Nano, 2013, 7, 9232-9240; (b) Z. Wu, T. Li, J. Li, W. Gao, T. Xu, C. Christianson and W. Gao, ACS Nano, 2014, 8, 12041.

23 B. Roy, A. Sahasrabudhe, B. Parasar, N. Ghosh, P. Panigrahi, A. Banerjee and S. Roy, J. Mol. Eng. Mater., 2014, 2, 1440006.

24 M. Ibele, T. E. Mallouk and A. Sen, Angew. Chem., Int. Ed., 2009, 48, 3308-3312.

25 Z. Wu, X. Lin, Y. Wu, T. Si, J. Sun and Q. He, ACS Nano, 2014, 8, 6097.

26 M. Guix, C. C. Mayorga-Martinez and A. Merkoçi, Chem. Rev., 2014, 114, 6285-6322.

27 S. Sattayasamitsathit, H. Kou, W. Gao, W. Thavarajah, K. Kaufmann, L. Zhang and J. Wang, Small, 2014, 10, 2830-2833.

28 T. R. Kline, W. F. Paxton, T. E. Mallouk and A. Sen, Angew. Chem., 2005, 117, 754-756.
29 L. Burlamacchi, G. Guarini and E. Tiezzi, Trans. Faraday Soc., 1969, 65, 496.

30 A. Muller, K. Das, E. Krickemyer and C. Kuhlmann, Inorg. Synth., 2004, 34, 191-200.

31 D. B. Allan, T. A. Caswell, N. C. Keim, Trackpyv0.2.ZENODO, 201410.5281/zenodo.9971.

32 A. Brown and W. Poon, Soft Matter, 2014, 10, 4016-4027.

33 W. Wang, T.-Y. Chiang, D. Velegol and T. E. Mallouk, J. Am. Chem. Soc., 2013, 135, 10557-10565.

34 L. L. del Mercato, M. Carraro, A. Zizzari, M. Bianco, R. Miglietta, V. Arima, I. Viola, C. Nobile, A. Sorarù and D. Vilona, Chem. - Eur. J., 2014, 20, 10910-10914.

35 J. Li, V. V. Singh, S. Sattayasamitsathit, J. Orozco, K. Kaufmann, R. Dong, W. Gao, B. Jurado-Sanchez, Y. Fedorak and J. Wang, ACS Nano, 2014, 8, 11118-11125.

36 Z. Wu, Y. Wu, W. He, X. Lin, J. Sun and Q. He, Angew. Chem., Int. Ed., 2013, 52, 7000. 